\title{
Prenatal exposure to perfluoroalkyl substances and anogenital distance at 3 months of age in a Danish mother-child cohort
}

\section{Citation}

Lind, Dorte Vesterholm, Lærke Priskorn, Tina Harmer Lassen, Flemming Nielsen, Henriette Boye Kyhl, David Møbjerg Kristensen, Henrik Thybo Christesen, Jan Steener Jørgensen, Philippe Grandjean, and Tina Kold Jensen. 2017. "Prenatal Exposure to Perfluoroalkyl Substances and Anogenital Distance at 3 Months of Age in a Danish Mother-Child Cohort." Reproductive Toxicology 68 (March): 200-206. doi:10.1016/j.reprotox.2016.08.019.

\section{Published Version}

10.1016/j.reprotox.2016.08.019

\section{Permanent link}

http://nrs.harvard.edu/urn-3:HUL.InstRepos:37221744

\section{Terms of Use}

This article was downloaded from Harvard University's DASH repository, and is made available under the terms and conditions applicable to Open Access Policy Articles, as set forth at http:// nrs.harvard.edu/urn-3:HUL.InstRepos:dash.current.terms-of-use\#OAP

\section{Share Your Story}

The Harvard community has made this article openly available.

Please share how this access benefits you. Submit a story.

Accessibility 


\section{Prenatal exposure to perfluoroalkyl substances and anogenital distance at $\mathbf{3}$ months of age in a Danish}

mother-child cohort

Dorte Vesterholm Lind ${ }^{1}$, Lærke Priskorn ${ }^{2}$, Tina Harmer Lassen ${ }^{2}$, Flemming Nielsen ${ }^{1}$, Henriette Boye Kyhl ${ }^{3,4}$,

David Møbjerg Kristensen ${ }^{5}$, Henrik Thybo Christesen ${ }^{3,7}$, Jan Steener Jørgensen ${ }^{6,7}$, Philippe Grandjean ${ }^{1,8}$, Tina

Kold Jensen ${ }^{1,3,4}$

${ }^{1}$ Department of Environmental Medicine, Institute of Public Health, University of Southern Denmark, Odense, Denmark.

${ }^{2}$ Rigshospitalet, Copenhagen University Hospital, Department of Growth and Reproduction, Blegdamsvej 9, 2100

Copenhagen, Denmark.

${ }^{3}$ Odense University Hospital, Hans Christian Andersen Children's Hospital , Sdr. Boulevard 29, 5000 Odense C, Denmark.

${ }^{4}$ Odense Patient data Exploratory Network (OPEN), University of Southern Denmark, Odense, Denmark.

${ }^{5}$ Laboratory of Genomic and Molecular Biomedicine, Department of Biology, University of Copenhagen,

Universitetsparken 13, DK-2100 Copenhagen, Denmark

${ }^{6}$ Odense University Hospital, Department of Obstetrics and Gynaecology, Sdr. Boulevard 29, 5000 Odense C, Denmark.

${ }^{7}$ Odense University Hospital, Institute for Clinical Research, Sdr. Boulevard 29, 5000 Odense C, Denmark

${ }^{8}$ Department of Environmental Health, Harvard T.H.Chan School of Public Health, Boston, MA 02215, USA.

Running title: Associations between PFASs and AGD

Keywords: perfluorinated compounds, endocrine disruption, reproduction, anogenital distance, birth weight, gestational length

\section{The authors declare they have no competing financial interests}

Corresponding author: Tina Kold Jensen, Department of Environmental Medicine, University of Southern

Denmark, Winsløwsparken 17A, 2.floor, 5000 Odense, Telephone +45 29464350, email:

tkjensen@health.sdu.dk

\section{Acknowledgements}

The technicians at Hans Christian Andersen's Children's Hospital are acknowledged for their careful

examination of the children. The study was funded by the Danish Environmental Protection Agency by way of the Centre on Endocrine Disruptors, the Danish Council for Strategic Research, Ronald McDonald Children 
Foundation, K. A. Rohde's and wife's Foundation, Odense University Hospital and Region of Southern Denmark, Danielsen Foundation, and Odense Patient data Exploratory Network (OPEN). 


\section{Abstract}

In the Odense child cohort, serum concentrations of perfluorooctanesulfonic acid (PFOS), perfluorooctanoic acid (PFOA), perfluorohexane sulfonic acid (PFHxS), perfluorononanoic acid (PFNA), and perfluorodecanoic acid (PFDA) were measured in 638 pregnant women. Birth weight, head and abdominal circumferences and gestational age were determined. Anogenital distance (AGD), the distance from the anus to the genital organs, and penile width were measured 3 months after expected date of birth in 511 children. PFOS, PFHxS, PFNA and PFDA were associated with a decreased AGD in girls ( $p$-trend $<0.05$ ) after adjusting for age and weight-for-age standard deviation score. PFOS in the highest quartile was associated with a $2.8 \mathrm{~mm}(95 \%$ confidence intervals $-4.5 ;-1.1)$ reduction in AGD in girls. No such tendencies were seen in boys. However, a tendency toward increased birth weight in girls and reduced birth weight in boys suggests that sex-dimorphic effects may occur from endocrine disrupting effects of these substances. 


\section{Introduction}

Perfluoroalkyl substances (PFASs) are synthetic chemicals produced in high quantities (Lindstrom, Strynar, \& Libelo, 2011). Their unique properties of high stability and low surface tension make them grease and water repellant and therefore suitable for use in a wide range of consumer products like food packaging, textiles, outdoor clothes, footwear and carpets; they are also used in paints, lubricants, waxes and in firefighting foams (Lindstrom et al., 2011; OECD, 2013). Perfluorooctanoic acid (PFOA) and perfluorooctane sulfonic acid (PFOS) have been most widely used; they are persistent in the environment and bio-accumulate (Lindstrom et al., 2011). They can be detected in human serum in Western populations, and they cross the placenta (Lindstrom et al., 2011; Needham et al., 2011). PFASs have a long elimination half-life -PFOS, 5.4 years and PFOA, 3.8 years (G. W. Olsen et al., 2007). In recent years, the use and production of PFOA and PFOS have been phased out due to the growing knowledge about their adverse health effects (Lindstrom et al., 2011). However, they have been replaced with the shorter and longer chained PFASs (e.g perfluorohexane sulfonic acid (PFHxS), perfluorononanoic acid (PFNA), and perfluorodecanoic acid (PFDA)) (Glynn et al., 2012) whose adverse effects have been studied less extensively.

Among other important adverse effects, PFASs may have endocrine disrupting properties (Lau et al., 2007). PFASs influence the expression of estrogen-responsive genes in animal studies (Benninghoff et al., 2011), and PFAS-induced changes in sex hormone biosynthesis have been reported in vitro (Du et al., 2013). PFASs have been shown to interfere with the estrogen receptor in human in vitro studies (Kjeldsen \& BonefeldJorgensen, 2013). PFAS exposure has been associated with fetal growth, and a recent review found that maternal exposure to PFOA and PFOS was associated with low birth weight, although the results were equivocal, perhaps due to differences in exposure and sex-dimorphic effects (Bach et al., 2014).

Anogenital distance (AGD; distance from anus to genitals) is routinely used in animal toxicology studies and is sensitive to anti-androgenic exposure. In rodents AGD has been shown to reflect the amount of androgen to which a male fetus is exposed in early development; males have longer AGD than females, and 
higher in utero androgen exposure results in longer AGD (Welsh et al., 2008). Numerous studies have shown that prenatal phthalate exposure shortens male AGD in rodents and humans (Foster, 2006; T. K. Jensen et al., 2016). High prenatal exposure to testosterone has also been associated with longer AGD in female rodent offspring, suggesting masculinization effects, and the changes were found to be indicators of permanent alteration in AGD in the adult female rodent (Hotchkiss et al. 2007). To our knowledge no human investigations have studied the effects of maternal PFAS exposure on AGD in the offspring, but PFASs exposure in wild male mink has been associated with shorter AGD (Persson \& Magnusson, 2014).

We therefore investigated the association between maternal PFAS exposure, including the less studied perfluorononanoic acid (PFNA), perfluorodecanoic acid (PFDA) and perfluorohexane sulfonic acid (PFHxS), and birth outcomes (birth weight, head and abdominal circumference and gestational length) and AGD at three months of age.

\section{Materials and Methods}

\subsection{Study population}

This study is based on data from the ongoing Odense Child Cohort (OCC). All women residing in the Municipality of Odense in the Region of Southern Denmark, who were pregnant between January 2010 and December 2012, were invited to participate. The pregnant women were recruited at a voluntary information meeting introducing the ultrasound examinations at Odense University Hospital (OUH) or at their first antenatal visit at gestational week 8 to 16 . Serum samples were collected at recruitment and stored in freezers at the Odense Patient data Explorative Network (OPEN). A total of 6,707 pregnant women were eligible, 4,017 were approached, and 2,874 (42.9\%) were successfully enrolled in the cohort with an informed consent form. The pregnant women filled in a questionnaire about general health and lifestyle. Participants were better educated, smoked less and were more often of Danish origin than non-participants (Kyhl et al., 2015). As of November 2014, 2,448 singleton children were active members of the cohort. 


\subsection{Birth outcome, AGD and covariates}

We obtained information from birth records about parity, maternal smoking, maternal pre-pregnancy BMI, gestational age (days) at birth and birth measures such as birth weight (grams), head circumference (cm), and abdominal circumference $(\mathrm{cm})$. Midwives assessed birth size right after parturition. Maternal ethnicity and education were obtained from a questionnaire completed in early pregnancy.

Three months after expected date of birth (median age 3.5 months, range 2.1 to 6.8 months) the children were invited to a clinical examination including measurements of length, weight and AGD. In boys, a short AGD was measured with a Vernier caliper from the center of the anus to the posterior base of the scrotum $\left(A G D_{a s}\right)$ and a long $A G D$ was measured from the center of the anus to the cephalad insertion of the penis $\left(A G D_{a p}\right)$. Penile width was also measured. Correspondingly, in girls a short AGD was measured from the center of the anus to the posterior fourchette $\left(A G D_{\text {af }}\right)$ and a long AGD from the center of the anus to the top of the clitoris $\left(A G D_{a c}\right)$. The genital measures were performed blindly and repeated three times in each child, and an arithmetic mean was calculated. All technicians attended training sessions and supervision. AGD measurements for the first 46 girls were excluded due to low accuracy. The coefficient of variation (CV) for AGDs was below $10 \%$ ( $3 \%$ on average for $A G D_{a s}, 2 \%$ for $A G D_{a p}, 4 \%$ for $A G D_{a f}$ and $3 \%$ for $A G D_{a c}$ ) for all the triplicate $A G D$ measurements, except for $A G D_{a f}$, in which two girls had CVs of 0.10 and 0.14 , respectively.

\subsection{PFAS measurements}

PFASs were measured in serum samples obtained at gestational week 5-12 (median 10 weeks) from a subset of 649 pregnant women. The first 200 samples were selected at random among women recruited in 2010, whereas an additional 449 were selected in 2011-2012 among those women who had available information from questionnaires, birth records and a clinical 3-month examination of the child. Three mothers from 2010 did not satisfy the latter criteria, and a total 638 children were therefore eligible for analyses of associations 
between birth outcomes and PFASs. The analysis of serum PFAS concentrations was performed by on-line solid phase extraction followed by liquid chromatography and triple quadropole mass spectrometry (LC-MS/MS). The quantified PFASs are comprised of perfluorooctanesulfonic acid (PFOS), perfluorooctanoic acid (PFOA), perfluorohexane sulfonic acid (PFHxS), perfluorononanoic acid (PFNA), and perfluorodecanoic acid (PFDA) (Haug, Thomsen, \& Becher, 2009). The Limit of Quantification (LOQ) was $0.03 \mathrm{ng} / \mathrm{mL}$ for all the reported compounds. Non-detectable concentrations were found only in a small number of samples and were replaced with half of the limit of quantification LOQ (i.e. $0.015 \mathrm{ng} / \mathrm{mL}$ ) (United States Environmental Protection Agency, 2000). Results from the first 200 samples and further descriptions of PFAS measurements have been reported elsewhere (Vorkamp et al., 2014).

\subsection{Ethics}

The study complied with the Declaration of Helsinki and was approved by the health research ethics committee system in Denmark (S-20090130) and the Danish Data Protection Agency (j.no. 2008-58-0035).

\subsection{Statistics}

PFASs distributions were skewed and were therefore divided into quartiles, but also entered as continuous variables after transformation by the natural logarithm to approach normality. Differences in serum-PFAS concentrations according to characteristics of the pregnant women were first tested by Kruskal Wallis test.

Multiple linear regression analysis was used to analyze the associations between maternal PFASs exposure and birth outcomes (birth weight, head and abdominal circumference and gestational age), AGD and penile width at 3-months of age adjusted for potential confounders. We tested for linear trends across PFASs quartiles in regression models by means of ordinal PFASs quartiles using integer values from 1 to 4 .

Confounders included in multivariable models were factors known a priori to be important predictors of birth outcomes or AGD. The AGD measurements, penile width and birth outcomes were left untransformed due to 
acceptable normal distributions of the residuals. The analyses of birth outcomes were adjusted for gestational age (days), parity (primiparous/multiparous), maternal smoking during pregnancy (yes/no), pre-pregnancy BMI $(<20,20-25,25+\mathrm{kg} / \mathrm{m} 2)$, maternal ethnicity (mother or maternal parents born in Western countries (yes/no), maternal education separated into primary (minimum $9^{\text {th }}$ grade), secondary (minimum high school or equivalent), and tertiary (minimum bachelor degree or equivalent). Maternal education and ethnicity were omitted from the final model, as they were not associated with any birth outcome. All analyses were tested for sex-dimorphic effects and stratified on child sex.

The multiple linear regression analyses of the associations between maternal PFASs exposure and AGD in the offspring were restricted to women of Western origin since AGD differed between children of Western and non-Western origin. Since AGD measurements vary with age and weight of the child, we constructed a measure of "post-conceptional age" at the time of AGD measurement as the sum of gestational age at birth (in days) and the age of the child at the AGD measurements (in days). Z-scores for the weight of child at the time of AGD measurement were calculated separately for boys and girls using all 2,041 singleton children who underwent the 3-month examination in Odense Child Cohort as reference. Analyses of associations between PFASs and AGD were adjusted for post-conceptional age, z-score for weight at 3 months, parity, maternal smoking, and pre-pregnancy BMI.

Residual plots were used to examine the model assumption of homogeneity of variances. Associations were considered statistically significant at the $p<0.05$ level and presented with $95 \%$ confidence intervals (Cls). All data analyses were performed using STATA.

\section{Results}

The mean age of the women at parturition was 30.7 years, $58 \%$ of the women were nulliparous, $3 \%$ were of non-Western origin and $3.2 \%$ smoked during pregnancy. Mean birth weight was 3,474g for girls and 3,600g for boys. Means of head circumference were 34.9 and $35.3 \mathrm{~cm}$, and mean abdominal circumferences were 33.3 
and $33.6 \mathrm{~cm}$ for girls and boys, respectively. Mean gestational length was 279.6 days for both boys and girls. Birth weight, abdominal and head circumference, sex, parity and age did not differ between women who had their serum PFASs measured $(N=638)$ and women who did not $(N=1,787)$. Fewer women with PFASs measurements smoked compared to those without PFASs measurements ( $3.2 \%$ vs. $5.3 \% p=0.03)$.

PFOS, PFOA, PFNA, PFDA were found in quantifiable concentrations in serum of all 649 women, while PFHxS was found in 99\% of the women (Table 1). The 200 women who gave birth in 2010 had higher concentrations of PFOA $(p<0.01)$, PFOS $(p=0.08)$ and PFNA $(p=0.17)$, but significantly lower concentrations of PFHxS $(p<0.01)$ and PFDA $(p<0.01)$ compared to the 449 women who gave birth in 2011-2012. Parity and age were negatively associated with PFASs. Women with high PFOA exposure were more often smokers and gave birth to children with low birth weight (Table 1).

Effects of PFASs exposure on birth weight were modified by sex ( $p$-value for interaction between sex and PFASs on birth weight; PFOA $p=0.26$, PFOS $p=0.06$, PFHxS $p=0.70$, PFNA $p=0.04$, PFDA $p=0.04$ ), and therefore all analyses were stratified by sex. No significant associations between maternal PFOA, PFOS, PFHxS and PFNAs exposure and birth weight or gestational length were found for boys or girls (Table S1). There was a tendency of maternal PFOS, PFNA and PFDA to be associated with reduced birth weight in boys and increased birth weight in girls (Figure S1) although this was not statistically significant. When PFASs exposures were entered as continuous variables no significant associations to birth weight, head and abdominal circumference were found (Table s1).

A total of 316 boys and 231 girls of mothers with available PFASs exposure had AGD measured. No consistent patterns were found between maternal PFASs exposure and AGD and penile width among boys (Table 2, Figure 1a). Among girls maternal exposure to all PFASs in the higher quartiles was associated with a decreased $A G D_{a c}$, $p$-trend for continuous PFAS exposure $<0.05$ for PFOS, PFHXS, PFNA and PFDA. Maternal PFOS exposure in the highest quartile was associated with $2.8 \mathrm{~mm}(-4.5 ;-1.1)$ reduction in AGD $_{\mathrm{ac}}$ (Table 2, Figure $1 \mathrm{~b}$ ). 


\section{Discussion}

In this prospective cohort study we found an association between maternal exposure to PFASs and reduced $A G D_{a c}$ in girls, but $A G D$ among boys was not affected. We did not find an association between maternal PFASs exposure and birth outcomes, however, a tendency towards lower birth weight among boys and higher birth weight among girls with high prenatal PFOS, PFNA and PFDA exposures was found. Thus, PFASs may have endocrine disrupting abilities as suggested by changes in AGD and a sex-dimorphic effect on birth weight.

Findings from other human studies of the adverse effects of prenatal PFASs exposure on birth outcomes (Geary W. Olsen, Butenhoff, \& Zobel, 2009) have been somewhat inconsistent. However, a recent review concluded that PFOA exposure was associated with a lower birth weight in eight published studies (Bach et al., 2014). Six out of eight published studies found that higher PFOS exposure was associated with lower birth weight. However, the associations were only statistically significant in a few studies (Bach et al., 2014). We found no association between PFOA and PFOS exposure and birth weight. However, our exposure levels were lower than in previous studies. The Danish National Birth Cohort, which included 1,400 pregnant women recruited from 1996-2002, reported average serum concentrations of PFOS and PFOA 3- to 4-fold higher than our study (PFOS mean, $35.5 \mathrm{ng} / \mathrm{mL}$; PFOA mean, $5.6 \mathrm{ng} / \mathrm{mL}$ ) (Fei, McLaughlin, Tarone, \& Olsen, 2007). They found a statistically significant association between prenatal PFOA exposure and low birth weight, but no effect of PFOS exposure (Fei et al., 2007). An American study conducted in areas with PFOA contaminated drinking water found no significant association between prenatal PFOA exposure and pregnancy outcome (preterm birth, gestational age, birth weight, still birth) even though the women had high PFOA exposure levels (PFOA mean, $31.0 \mathrm{ng} / \mathrm{mL}$ )(Darrow, Stein, \& Steenland, 2013; Savitz et al., 2012). Similarly, two Canadian studies did not find any associations between PFOA, PFOS, PFHxS and PFNA exposure and birth weight (Hamm, Cherry, Chan, Martin, \& Burstyn, 2010; Monroy et al., 2008). Although a cohort study of 429 pregnant women from Taiwan found a decreased birth weight and head circumference at increasing PFOS exposure, no associations were observed for PFOA and PFNA (Chen et al., 2012). To our knowledge, no other 
human studies have examined the associations between prenatal PFNA and PFDA exposures and birth outcomes. In addition, most previous studies did not stratify their findings by sex, as suggested by Bach et al., thus possibly overlooking potential sex-dimorphic effects, as we found a tendency for girls to have increased birth weight and boys to have a reduced birth weight.

AGD is routinely used in animal toxicology studies and is sensitive to anti-androgenic exposure. In rodents AGD has been shown to reflect the amount of androgen to which a male fetus is exposed in early development; males have longer AGD than females, and higher in utero androgen exposure results in longer AGD. Several studies have found that exposure to chemicals with anti-androgenic action e.g. phthalates reduce AGD in male infants (Bornehag et al., 2015; T. K. Jensen et al., 2016; Swan et al., 2015), and Huang found a negative association between prenatal phthalate exposure and AGD in newborn girls (Huang, Kuo, Chou, Lin, \& Lee, 2009). To our knowledge, no other studies have explored a potential association between PFASs and AGD in humans, but one study in wild mink found a decreased AGD in male mink exposed to PFASs (Persson \& Magnusson, 2014), a finding which we could not replicate. They did not, however, study female minks.

PFASs may have endocrine disrupting properties (Lau et al., 2007). Some studies have suggested that PFASs antagonize the androgen receptor activity (Du et al., 2013); the effects on the male reproductive system include increased germ cell apoptosis (Feng et al., 2009) and decreased weight of the reproductive organs (Shi et al., 2007). In addition, PFASs seem to affect testosterone concentrations by decreasing the expression of genes involved in steroid biosynthesis (Shi et al., 2007; Du et al., 2013). PFOA, PFOS and PFDA affect both estradiol and testosterone (Biegel, Liu, Hurtt, \& Cook, 1995; Bookstaff, Moore, Ingall, \& Peterson, 1990; A. A. Jensen \& Leffers, 2008; Lau et al., 2007; Ye, Su, \& Ge, 2011). The effects on the female reproductive system have been investigated less frequently, but one study suggested that estrous cyclicity may be disrupted (Austin et al., 2003). If androgens are lowered this may reduce female AGD. 
Our study has several strengths: it is large and comprised of population-based mother-child pairs, birth outcomes were well reported, and AGDs were measured three times by trained technicians. However, only $42 \%$ of the eligible women participated in this study and participants were better educated than nonparticipants, making selection bias possible. However, the pregnant women had no knowledge of their PFASs exposure or the birth outcome or AGD of their child at enrollment, and it is therefore unlikely that PFASs exposure was associated with willingness to participate. In addition, we compared women across a range of PFASs exposures, so whether they represented the general population or not is therefore of less importance. We adjusted for relevant confounders, but we cannot exclude the possibility of residual confounding by other factors associated with PFASs exposure and growth measures, such as e.g. co-exposure to other environmental chemicals, lifestyle, or health behavior. We performed a large number of statistical analyses and some of our results may therefore be chance findings due to multiple testing. However, the reduction in female AGD was seen across all exposure categories and in all five PFAS compounds.

Shorter AGD has been associated with hypospadias and cryptorchidism in human males (Hsieh, Breyer, Eisenberg, \& Baskin, 2008; Hsieh et al., 2012; Thankamony et al., 2014), as well as reduced serum-testosterone concentrations and reduced semen quality (Eisenberg, Hsieh, Walters, Krasnow, \& Lipshultz, 2011; Eisenberg, Jensen, Walters, Skakkebaek, \& Lipshultz, 2012; Mendiola, Stahlhut, Jorgensen, Liu, \& Swan, 2011). AGD in males may be used as a marker of testicular dysgenesis syndrome (TDS), which suggests that the increased incidence of reproductive problems such as hypospadias, cryptorchidism, testis cancer and poor semen quality are symptoms of a shared underlying fetal testicular dysgenesis resulting from a disturbance in the Sertoli cell and Leydig cell differentiation during fetal life, leading to impaired testosterone production and decreased virilization. Less is known about AGD in females and their reproductive system characteristics (Mendiola et al., 2012). However, a study found positive associations between AGD and follicular number in women (Mendiola et al., 2012). We found shorter AGD in girls prenatally exposed to PFASs. The long term effects of reduced AGD on reproductive health in girls have not been studied but need to be addressed in future studies. 


\section{Conclusion}

In conclusion, we found that maternal PFASs exposure was associated with reduced AGD in girls, although no clear association was seen in boys. In addition, no significant association between maternal PFASs exposure and birth outcomes were observed, however, a sex-dimorphism pattern was suggested with a tendency towards lower birth weight among boys and higher birth weight among girls, with high prenatal PFOS, PFNA and PFDA exposures found. Thus, PFASs may have endocrine disrupting abilities as suggested by changes in AGD and a sex-dimorphic effect on birth weight. To our knowledge this study is the first to report a possible association between PFASs and female AGD, and no clear mechanisms of action can be suggested. Future studies to elucidate this are urgently needed. 


\section{References}

Austin, M. E., Kasturi, B. S., Barber, M., Kannan, K., MohanKumar, P. S., \& MohanKumar, S. M. (2003). Neuroendocrine effects of perfluorooctane sulfonate in rats. Environ Health Perspect, 111(12), 14851489.

Bach, C. C., Bech, B. H., Brix, N., Nohr, E. A., Bonde, J. P., \& Henriksen, T. B. (2014). Perfluoroalkyl and polyfluoroalkyl substances and human fetal growth: A systematic review. Crit Rev Toxicol, 1-15.

Benninghoff, A. D., Bisson, W. H., Koch, D. C., Ehresman, D. J., Kolluri, S. K., \& Williams, D. E. (2011). Estrogenlike activity of perfluoroalkyl acids in vivo and interaction with human and rainbow trout estrogen receptors in vitro. Toxicol Sci, 120(1), 42-58.

Biegel, L. B., Liu, R. C., Hurtt, M. E., \& Cook, J. C. (1995). Effects of ammonium perfluorooctanoate on Leydig cell function: in vitro, in vivo, and ex vivo studies. Toxicol Appl Pharmacol, 134(1), 18-25.

Bookstaff, R. C., Moore, R. W., Ingall, G. B., \& Peterson, R. E. (1990). Androgenic deficiency in male rats treated with perfluorodecanoic acid. Toxicol Appl Pharmacol, 104(2), 322-333.

Bornehag, C. G., Carlstedt, F., Jonsson, B. A., Lindh, C. H., Jensen, T. K., Bodin, A., et al. (2015). Prenatal phthalate exposures and anogenital distance in Swedish boys. Environ Health Perspect, 123(1), 101107.

Chen, M. H., Ha, E. H., Wen, T. W., Su, Y. N., Lien, G. W., Chen, C. Y., et al. (2012). Perfluorinated compounds in umbilical cord blood and adverse birth outcomes. PLoS One, 7(8), e42474.

Darrow, L. A., Stein, C. R., \& Steenland, K. (2013). Serum perfluorooctanoic acid and perfluorooctane sulfonate concentrations in relation to birth outcomes in the Mid-Ohio Valley, 2005-2010. Environ Health Perspect, 121(10), 1207-1213.

Du, G., Huang, H., Hu, J., Qin, Y., Wu, D., Song, L., et al. (2013). Endocrine-related effects of perfluorooctanoic acid (PFOA) in zebrafish, H295R steroidogenesis and receptor reporter gene assays. Chemosphere, 91(8), 1099-1106.

Eisenberg, M. L., Hsieh, M. H., Walters, R. C., Krasnow, R., \& Lipshultz, L. I. (2011). The relationship between anogenital distance, fatherhood, and fertility in adult men. PLoS One, 6(5), e18973.

Eisenberg, M. L., Jensen, T. K., Walters, R. C., Skakkebaek, N. E., \& Lipshultz, L. I. (2012). The relationship between anogenital distance and reproductive hormone levels in adult men. J Urol, 187(2), 594-598.

Fei, C., McLaughlin, J. K., Tarone, R. E., \& Olsen, J. (2007). Perfluorinated chemicals and fetal growth: a study within the Danish National Birth Cohort. Environ Health Perspect, 115(11), 1677-1682.

Foster, P. M. (2006). Disruption of reproductive development in male rat offspring following in utero exposure to phthalate esters. [Review]. Int J Androl, 29(1), 140-147; discussion 181-145.

Glynn, A., Berger, U., Bignert, A., Ullah, S., Aune, M., Lignell, S., et al. (2012). Perfluorinated alkyl acids in blood serum from primiparous women in Sweden: serial sampling during pregnancy and nursing, and temporal trends 1996-2010. Environ Sci Technol, 46(16), 9071-9079.

Hamm, M. P., Cherry, N. M., Chan, E., Martin, J. W., \& Burstyn, I. (2010). Maternal exposure to perfluorinated acids and fetal growth. J Expo Sci Environ Epidemiol, 20(7), 589-597.

Haug, L. S., Thomsen, C., \& Becher, G. (2009). A sensitive method for determination of a broad range of perfluorinated compounds in serum suitable for large-scale human biomonitoring. J Chromatogr A, 1216(3), 385-393.

Hsieh, M. H., Breyer, B. N., Eisenberg, M. L., \& Baskin, L. S. (2008). Associations among hypospadias, cryptorchidism, anogenital distance, and endocrine disruption. Curr Urol Rep, 9(2), 137-142.

Hsieh, M. H., Eisenberg, M. L., Hittelman, A. B., Wilson, J. M., Tasian, G. E., \& Baskin, L. S. (2012). Caucasian male infants and boys with hypospadias exhibit reduced anogenital distance. Hum Reprod.

Huang, P. C., Kuo, P. L., Chou, Y. Y., Lin, S. J., \& Lee, C. C. (2009). Association between prenatal exposure to phthalates and the health of newborns. Environ Int, 35(1), 14-20. 
Jensen, T. K., Frederiksen, H., Kyhl, H. B., Lassen, T. H., Swan, S. H., Bornehag, C. G., et al. (2016). Prenatal Exposure to Phthalates and Anogenital Distance in Male Infants from a Low-Exposed Danish Cohort (2010-2012). Environ Health Perspect, 124(7), 1107-1113.

Jensen, A. A., \& Leffers, H. (2008). Emerging endocrine disrupters: perfluoroalkylated substances. [Review]. Int J Androl, 31(2), 161-169.

Kjeldsen, L. S., \& Bonefeld-Jorgensen, E. C. (2013). Perfluorinated compounds affect the function of sex hormone receptors. Environ Sci Pollut Res Int, 20(11), 8031-8044.

Kyhl, H. B., Jensen, T. K., Barington, T., Buhl, S., Norberg, L. A., Jorgensen, J. S., et al. (2015). The Odense Child Cohort: Aims, Design, and Cohort Profile. Paediatr Perinat Epidemiol.

Lau, C., Anitole, K., Hodes, C., Lai, D., Pfahles-Hutchens, A., \& Seed, J. (2007). Perfluoroalkyl acids: a review of monitoring and toxicological findings. [Review]. Toxicol Sci, 99(2), 366-394.

Lindstrom, A. B., Strynar, M. J., \& Libelo, E. L. (2011). Polyfluorinated compounds: past, present, and future. Environ Sci Technol, 45(19), 7954-7961.

Mendiola, J., Roca, M., Minguez-Alarcon, L., Mira-Escolano, M. P., Lopez-Espin, J. J., Barrett, E. S., et al. (2012). Anogenital distance is related to ovarian follicular number in young Spanish women: a cross-sectional study. Environ Health, 11, 90.

Mendiola, J., Stahlhut, R. W., Jorgensen, N., Liu, F., \& Swan, S. H. (2011). Shorter anogenital distance predicts poorer semen quality in young men in Rochester, New York. Environ Health Perspect, 119(7), 958-963.

Monroy, R., Morrison, K., Teo, K., Atkinson, S., Kubwabo, C., Stewart, B., et al. (2008). Serum levels of perfluoroalkyl compounds in human maternal and umbilical cord blood samples. Environ Res, 108(1), 56-62.

Needham, L. L., Grandjean, P., Heinzow, B., Jorgensen, P. J., Nielsen, F., Patterson, D. G., Jr., et al. (2011). Partition of environmental chemicals between maternal and fetal blood and tissues. Environ Sci Technol, 45(3), 1121-1126.

OECD. (2013). OECD/UNEP Global PFC Group, Synthesis paper on per- and polufluorinated chemicals (PFCs): Environment, Health and Safety, Environment Directorate, OECD.

Olsen, G. W., Burris, J. M., Ehresman, D. J., Froehlich, J. W., Seacat, A. M., Butenhoff, J. L., et al. (2007). Half-life of serum elimination of perfluorooctanesulfonate,perfluorohexanesulfonate, and perfluorooctanoate in retired fluorochemical production workers. Environ Health Perspect, 115(9), 1298-1305.

Olsen, G. W., Butenhoff, J. L., \& Zobel, L. R. (2009). Perfluoroalkyl chemicals and human fetal development: An epidemiologic review with clinical and toxicological perspectives. Reproductive Toxicology, 27(3-4), 212-230.

Persson, S., \& Magnusson, U. (2014). Environmental pollutants and alterations in the reproductive system in wild male mink (Neovison vison) from Sweden. Chemosphere, 120C, 237-245.

Savitz, D. A., Stein, C. R., Bartell, S. M., Elston, B., Gong, J., Shin, H. M., et al. (2012). Perfluorooctanoic acid exposure and pregnancy outcome in a highly exposed community. Epidemiology, 23(3), 386-392.

Swan, S. H., Sathyanarayana, S., Barrett, E. S., Janssen, S., Liu, F., Nguyen, R. H., et al. (2015). First trimester phthalate exposure and anogenital distance in newborns. Hum Reprod, 30(4), 963-972.

Thankamony, A., Lek, N., Carroll, D., Williams, M., Dunger, D. B., Acerini, C. L., et al. (2014). Anogenital distance and penile length in infants with hypospadias or cryptorchidism: comparison with normative data. Environ Health Perspect, 122(2), 207-211.

Vorkamp, K., Nielsen, F., Kyhl, H. B., Husby, S., Nielsen, L. B., Barington, T., et al. (2014). Polybrominated diphenyl ethers and perfluoroalkyl substances in serum of pregnant women: levels, correlations, and potential health implications. Arch Environ Contam Toxicol, 67(1), 9-20.

Welsh, M., Saunders, P. T., Fisken, M., Scott, H. M., Hutchison, G. R., Smith, L. B., et al. (2008). Identification in rats of a programming window for reproductive tract masculinization, disruption of which leads to hypospadias and cryptorchidism. J Clin Invest, 118(4), 1479-1490. 
Ye, L., Su, Z. J., \& Ge, R. S. (2011). Inhibitors of testosterone biosynthetic and metabolic activation enzymes. Molecules, 16(12), 9983-10001. 